\title{
ESTIMATIVA DA VULNERABILIDADE DOS SOLOS À EROSÃO HÍDRICA NA BACIA HIDROGRÁFICA DO RIO CERVO - MG.
}

\section{ESTIMATING SOIL EROSION VULNERABILITY IN THE CERVO RIVER BASIN - MG.

\author{
Jéssica Assaid Martins RODRIGUES, Carlos Rogério de MELLO, Marcelo Ribeiro \\ VIOLA, Maykmiller Carvalho RODRIGUES
}

Universidade Federal de Lavras, Departamento de Engenharia. Lavras, MG. Endereços eletrônicos: je_assaid@yahoo.com.br; crmello@deg.ufla.br; marcelo.viola@deg.ufla.br; maykimiller.rodrigues@deg.ufla.br

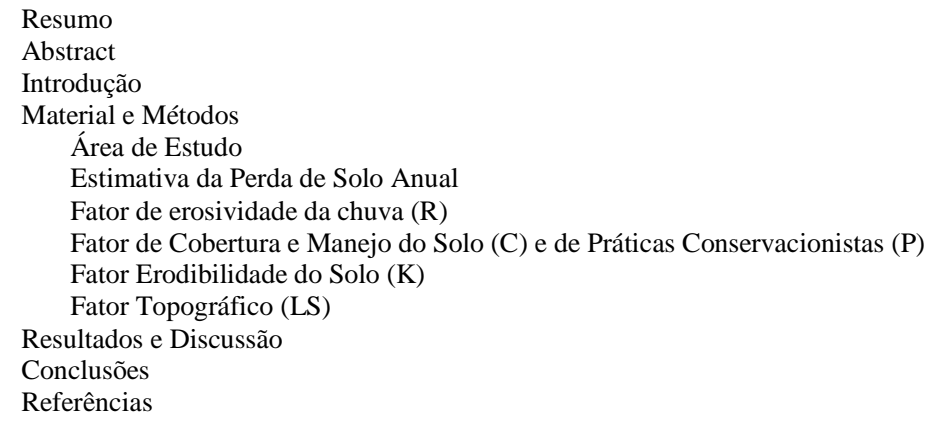

\begin{abstract}
RESUMO - Objetivou-se no presente trabalho estimar a distribuição espacial do potencial anual médio de perda de solos na bacia hidrográfica do Rio Cervo, MG, a fim de identificar as áreas mais susceptíveis à erosão hídrica e subsidiar possíveis alterações na gestão dos recursos naturais. Para isso foi aplicado o modelo RUSLE (Revised Universal Soil Loss Equation) implementado em um ambiente SIG. Os fatores utilizados pelo modelo foram gerados em formato raster e combinados por meio de álgebra de mapas. O fator de erosividade da chuva (R) foi obtido com base em um modelo geográfico multivariado desenvolvido para o Sudeste do Brasil; o fator de cobertura e manejo do solo (C), foi derivado do mapa de uso do solo confeccionado a partir de imagens Rapideye referentes ao ano de 2015; o fator erodibilidade do solo (K), foi obtido pelo mapa de solos, e o fator topográfico (LS), pelo MDE (Modelo digital de elevação). Os resultados mostraram que 40,59\% dos solos da bacia hidrográfica apresentam perdas superiores a $10 \mathrm{t} \mathrm{ha}^{-1} \mathrm{ano}^{-1}$, com predomínio da classe de vulnerabilidade "muito alta"; e $41 \%$ da área total apresenta perda de solo superior à taxa tolerável. A partir da distribuição espacial das nascentes, verificou-se que 35,6\% dessas estão localizadas em áreas que apresentam perdas de solo superiores a $15 \mathrm{t} \mathrm{ha}^{-1}$ ano $^{-1}$, este resultado é decorrente do predomínio de solo exposto, pastagem e agricultura combinado a regiões de Neossolo Litólico. Desta forma, verificou-se a necessidade de implementar práticas de manejo da cobertura e conservação do solo a fim de reduzir a vulnerabilidade à erosão hídrica na bacia hidrográfica do Rio Cervo.

Palavras-chave: erosão do solo, RUSLE, gestão de bacias hidrográficas, SIG.
\end{abstract}

\begin{abstract}
The objective of this paper is to estimate the spatial distribution of the average potential annual soil loss in the Cervo River basin, MG, in order to identify the areas most susceptible to water erosion and support possible changes in the natural resources management. By this way, it was applied the RUSLE model (Revised Universal Soil Loss Equation) implemented in a GIS. The factors used in the model were generated in raster format and combined by algebra of maps. The rainfall erosivity factor (R) was obtained based on a geographical multivariate model developed for the Southeast of Brazil; the coverage and management soil factor (C), was derived from land use map made from RapidEye images of 2015; the soil erodibility factor (K), was obtained by the soil map, and the topographic factor (LS), by the DEM (Digital Model Lifting). The results showed that $40.59 \%$ of the basin soils have lost more than $10 \mathrm{t} \mathrm{ha}^{-1} \mathrm{yr}^{-1}$, with predominance "high" class of vulnerability; and $41 \%$ of the total area showed the soil loss exceeding the tolerable rate. By the spatial distribution of sources, it was found that $35.6 \%$ of these are located in areas with more than $15 \mathrm{t} \mathrm{ha}^{-1} \mathrm{yr}^{-1}$ of soil loss, this result is due to the exposed soil predominance, grazing and agriculture combined with the Udorthent Neossolo regions. Thus, it needs to implement some practices of land cover management and soil conservation in order to reduce the vulnerability by water erosion in the Cervo River basin.
\end{abstract}

Keywords: soil erosion, RUSLE, basin management, GIS.

\section{INTRODUÇÃO}

A erosão do solo consiste em um dos problemas mais iminentes da humanidade, responsável pelo decrescimento da qualidade e produtividade do solo, pelo esgotamento das terras agricultáveis, redução da infiltração, aumento do escoamento superficial e pela deterioração da qualidade da água. Além disso, a produção de sedimentos resulta no assoreamento de rios e reservatórios, comprometendo a geração de energia elétrica bem como causando inundações em áreas urbanas (Capoane, 2013).
A degradação do solo é resultante de uma combinação de mudanças no seu uso, intensificação da agricultura e chuvas intensas. Para minimizar e mitigar esse processo, deve-se promover a utilização racional da terra por meio da conservação e gestão adequada das bacias hidrográficas, tendo-se como base informações sobre o potencial erosivo da bacia e sua capacidade de produção de sedimentos (Pandey et al., 2007).

Como as mensurações diretas da erosão em grandes escalas são inviáveis devido a 
restrições metodológicas e financeiras, uma intervenção eficiente no controle da erosão é a aplicação de modelos de predição da erosão, os quais consideram os fatores passivos e ativos da erosão, associados ao sistema de informações geográficas (SIGs) (Mitasova et al., 2001; Panagos et al., 2015).

Existem diversos modelos matemáticos que identificam as áreas mais vulneráveis à erosão hídrica, avaliam os fatores que afetam a perda de solo e a sua distribuição espacial e simulam possíveis cenários futuros de gestão (Shiferaw, 2011; Ayer et al., 2015).

O modelo RUSLE (Revised Universal Soil Loss Equation) (Renard et al., 1997), versão revisada do modelo USLE (Universal Soil Loss Equation), é considerado um modelo simples de predição da erosão que permite estimar a erosão hídrica com base na interação entre os fatores erosividade da chuva, erodibilidade do solo, influências do relevo, da cobertura e do manejo do solo, a partir da utilização de ferramentas SIG. Devido à sua maior simplicidade e disponibilidade dos dados de entrada, o modelo RUSLE ainda é o mais amplamente utilizado para estimar a perda de solo média anual em grandes escalas, apresentando uma acurácia aceitável (Kinnell, 2010; Oliveira et al., 2014; Panagos et al., 2015).

O sul do estado de Minas Gerais está entre as regiões com maior destaque no cenário nacional, sendo a principal produtora mundial de café e uma das principais na produção de leite no Brasil, além de atuar como importante fornecedora de energia para as regiões metropolitanas brasileiras, como Belo Horizonte, Rio de Janeiro e São Paulo (FJP, 2009; Ayer et al., 2015).

No entanto, nessa região existem poucos estudos que tenham detalhado espacialmente a vulnerabilidade dos solos à erosão e relacionam com os sistemas de uso do solo. Neste sentido, o objetivo deste trabalho foi aplicar o modelo RUSLE (Revised Universal Soil Loss Equation) para estimar a distribuição espacial do potencial anual médio de perda de solos na bacia hidrográfica do Rio Cervo, afluente direto do reservatório de Furnas, a fim de identificar as áreas mais susceptíveis à erosão hídrica e subsidiar possíveis alterações na gestão dos recursos naturais na bacia, visando minimizar a perda de solo.

\section{Área de Estudo}

\section{MATERIAL E MÉTODOS}

A bacia hidrográfica do Rio Cervo está localizada na região sul do estado de Minas Gerais, entre as latitudes $21^{\circ} 14^{\prime} \mathrm{S}$ e $21^{\circ} 38^{\prime} \mathrm{S}$ e as longitudes $44^{\circ} 55^{\prime} \mathrm{W}$ e $45^{\circ} 23^{\prime} \mathrm{W}$, com altitudes variando de 808 a 1509 metros, e área de drenagem de $1054 \mathrm{~km}^{2}$, considerando como seção fluviométrica a Estação Fluviométrica UHE Furnas Rio do Cervo (Código na Agência Nacional de Águas - ANA: 61176000), controlada pela Furnas Centrais Elétricas S.A (Figura 1).

A bacia faz parte da Bacia do Rio Grande e é afluente do reservatório da Usina Hidrelétrica de Furnas, pertencendo a Unidade de Planejamento e Gestão e Recursos Hídricos GD2. De acordo com a classificação de Köppen, o clima é temperado úmido com inverno seco e verão quente - Cwa (Sá Júnior 2009), com precipitação média anual de $1400 \mathrm{~mm}$.

\section{Estimativa da Perda de Solo Anual}

A Equação Universal de Perda de Solo Revisada (RUSLE) é um modelo paramétrico que foi testado e validado em diversas condições de solo, clima e manejo (Renard et al., 1997). A RUSLE implementada em um ambiente SIG foi utilizada para estimar a perda média anual de solo na bacia do Rio Cervo. Essa equação é baseada numa função de cinco principais fatores que afetam a erosão do solo (Eq. 1), gerados individualmente na forma de mapas trabalhados em formato raster, em uma resolução espacial de 30 metros, os quais foram combinados pixel a pixel, a partir da aplicação de álgebra de mapas (Bonilla et al., 2010; Shiferaw, 2011; Ozsoy et al., 2012) (Figura 2).

$$
\mathrm{A}=\mathrm{R} \times \mathrm{K} \times \mathrm{LS} \times \mathrm{C} \times \mathrm{P}
$$

Sendo, A a perda média anual de solo por unidade de área (t.ha $\left.{ }^{-1} \cdot \mathrm{ano}^{-1}\right), \mathrm{R}$ é o fator de erosividade da chuva (MJ.mm.ha- ${ }^{-1} \cdot \mathrm{h}^{-1} \cdot \mathrm{ano}^{-1}$ ), $\mathrm{K}$ é o fator de erodibilidade do solo (t.h. $\mathrm{MJ}^{-1} \cdot \mathrm{mm}^{-1}$ ), LS é o fator topográfico (adimensional), $\mathrm{C}$ é o fator de cobertura e manejo do solo (adimensional), e $\mathrm{P}$ é o fator de práticas conservacionistas (adimensional). Os procedimentos para calcular os fatores individuais são descritos a seguir. 


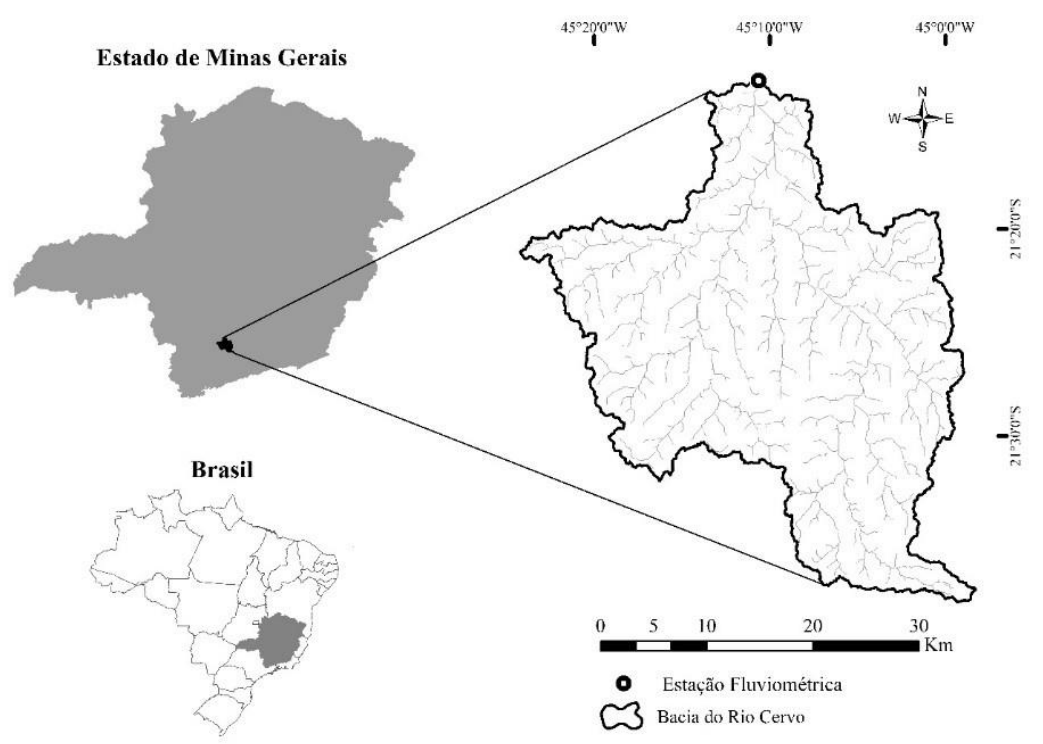

Figura 1 - Localização geográfica da bacia hidrográfica do Rio Cervo, Minas Gerais.

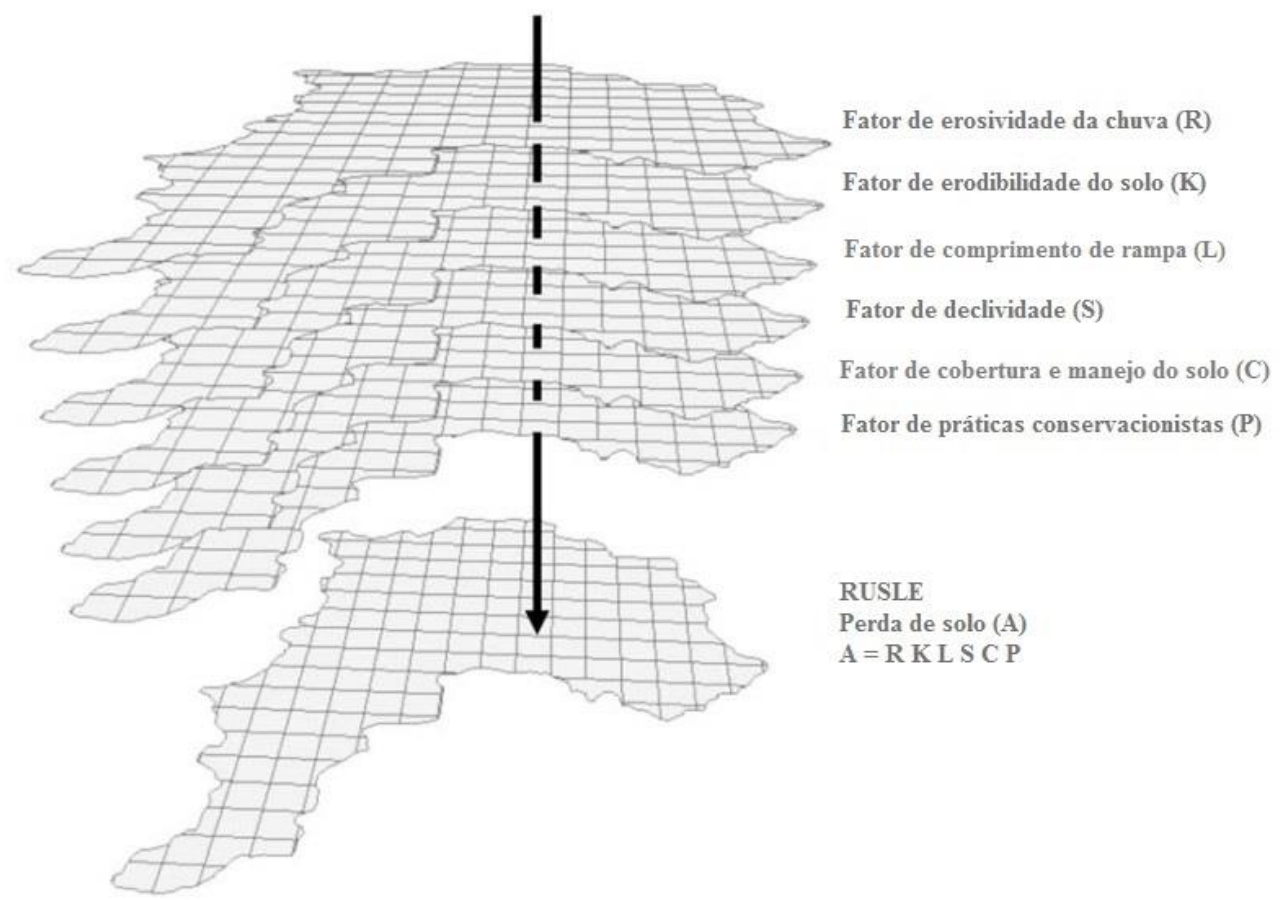

Figura 2 - Equação Universal de Perda de Solo Revisada (RUSLE) implementada em um SIG.

Fonte: Adaptado de (Bonilla et al., 2010).

\section{Fator de Erosividade da Chuva $(R)$}

$O$ fator de erosividade (R) representa o potencial natural da chuva de provocar erosão. É definido como o produto entre a energia cinética da chuva e sua intensidade máxima consecutiva com duração de 30 minutos (EI30) (Wischmeier \& Smith, 1978). Contudo, para aplicação da RUSLE, este fator é entendido como o valor médio anual da erosividade, conhecido como Fator R, para o qual são necessários no mínimo 15 anos de observação de dados de EI30, normalmente oriundos de pluviogramas. Devido à baixa disponibilidade tanto espacial quanto de série histórica de precipitação baseada pluviograma, normalmente adotam-se estimativas do fator $\mathrm{R}$, tendo-se como referência, estações cuja relação entre o fator $\mathrm{R}$ e a precipitação média mensal (ou o Índice Modificado de Fournier) esteja disponível.

Neste estudo, o mapa do fator $\mathrm{R}$ foi desenvolvido com base em um modelo 
geográfico multivariado desenvolvido por Mello et al. (2013) para a região Sudeste do Brasil, no qual estima-se o fator $\mathrm{R}$ em função da latitude, longitude e a altitude de cada uma das células da bacia hidrográfica, extraídas a partir modelo digital de elevação (MDE). O modelo para estimar o fator $\mathrm{R}$ médio anual para o Sudeste do Brasil é dado por:

$\mathbf{R}=-399433+420.49 \times \mathrm{A}-78,296 \times \mathrm{LA}-$ $0.01784 \times \mathrm{A}^{2}-1594.04 \times \mathrm{LA}^{2}+195.84 \times \mathrm{LO}^{2}$ $+17.77 \times$ LO $\times$ A $-1716.27 \times$ LA $\times$ LO + $0.1851 \times \mathrm{LO}^{2} \times \mathrm{A}+0.00001002 \times \mathrm{LO}^{2} \times \mathrm{A}^{2}+$ $1,389 \times \mathrm{LO}^{2} \times \mathrm{LA}^{2}+0.01364 \times \mathrm{LA}^{2} \times \mathrm{LO}^{3}$

Sendo, $\mathrm{R}$ é o fator de erosividade da chuva (MJ.mm.ha ${ }^{-1} \cdot h^{-1} \cdot$ ano $^{-1}$ ), A é a altitude (m); LA é a latitude e LO é a longitude, ambos em graus decimais negativos.

\section{Fator de Cobertura e Manejo do Solo (C) e de Práticas Conservacionistas $(\mathrm{P})$}

$\mathrm{O}$ fator de cobertura e manejo do solo (C) representa a relação entre a perda de solo ocorrida em uma área coberta e sob um manejo em particular e a perda ocorrida em uma área equivalente, considerando o mesmo tipo de solo, declive e erosividade da chuva, porém, com o solo continuamente descoberto por 2 anos. $\mathrm{O}$ fator $\mathrm{C}$ varia de 0 a 1 , onde valores próximos de 1 representam áreas com cobertura vegetal quase nula, e, portanto, mais vulneráveis à erosão hídrica. Esse é um fator fundamental para aplicação da RUSLE, uma vez que representa as condições que podem ser facilmente alteradas para conter a erosão do solo. Neste trabalho, o mapa de cobertura e manejo do solo foi desenvolvido a partir de imagens do sensor multiespectral "Rapideye", com resolução espacial de 5 metros e referentes ao ano de 2015, disponibilizadas a partir Ministério do Meio Ambiente. Foram consideradas oito classes de uso do solo, a saber: área urbana, água, afloramento rochoso, agricultura, eucalipto, pastagem, mata e solo exposto.

A classificação do uso e manejo do solo foi realizada em duas etapas. Primeiramente, foi gerada uma classificação supervisionada, utilizando o algoritmo de classificação de imagens Support Vector Machine - SVM, com orientação baseada em objetos; na sequência, foi realizada uma reclassificação por meio da edição manual das classes de uso do solo, com o objetivo de corrigir eventuais falhas do algoritmo e incrementar a acurácia do mapeamento. Os valores de $\mathrm{C}$ empregados a cada classe foram obtidos com base em estudos publicados para os mesmos usos, conforme Tabela 1.

$\mathrm{O}$ fator de práticas conservacionistas $(\mathrm{P})$ representa à relação entre a perda de solo em uma área sob dada prática de suporte e a perda de solo em uma área equivalente, sem nenhuma prática. Dentre as práticas conservacionistas de suporte, pode-se citar o cultivo em contorno, o cultivo em faixas com rotação de culturas e o terraceamento, as quais controlam a erosão por meio da diminuição da velocidade e capacidade de transporte da enxurrada (Amaral, 2006). Como na maior parte da área de estudo não existe a adoção de práticas conservacionistas de suporte a erosão, o fator $\mathrm{P}$ foi considerado 1 para toda a bacia, conforme procedimento também adotado por Beskow et al., 2009; Oliveira et al., 2014; Dias \& Silva, 2015.

Tabela 1 - Valores do fator C para as condições de cobertura e uso do solo na bacia.

\begin{tabular}{c|c|c}
\hline USO DO SOLO & C & FONTE \\
\hline Área Urbana & 0 & - \\
\hline Água & 0 & - \\
\hline Afloramento Rochoso & 0 & - \\
\hline Agricultura & 0,29 & (Ruhoff et al., 2006) \\
\hline Eucalipto & 0,3 & (Martins et al., 2010) \\
\hline Pastagem & 0,09 & (Ozsoy et al., 2012) \\
\hline Mata & 0,01 & (Oliveira et al., 2014) \\
\hline Solo Exposto & 1 & - \\
\hline
\end{tabular}

\section{Fator Erodibilidade do Solo (K)}

Diferentes tipos de solos são naturalmente mais resistentes ou susceptíveis a erosão do que outros. A erodibilidade do solo (Fator K) é definida como a taxa de perda de solo por unidade de erosividade da chuva, de um solo sem uso e manejo (solo exposto) e representa a susceptibilidade intrínseca do solo à erosão hídrica (Wischmeier \& Smith, 1978). Depende de fatores tais como textura do solo, teor de matéria 
orgânica, coesão, classe de permeabilidade, dentre outros (Ozsoy et al., 2012).

Como os solos da bacia do Rio Cervo (Figura 3) foram devidamente estudados por Feam (2010) para compor o Mapa de Solos do Estado de
Minas Gerais, na escala de 1:500.000, esta foi a base para a aquisição do fator $\mathrm{K}$ adotada neste estudo. Os valores do fator $\mathrm{K}$ foram extraídos de diferentes literaturas que estudaram os mesmos tipos de solos em questão (Tabela 2).

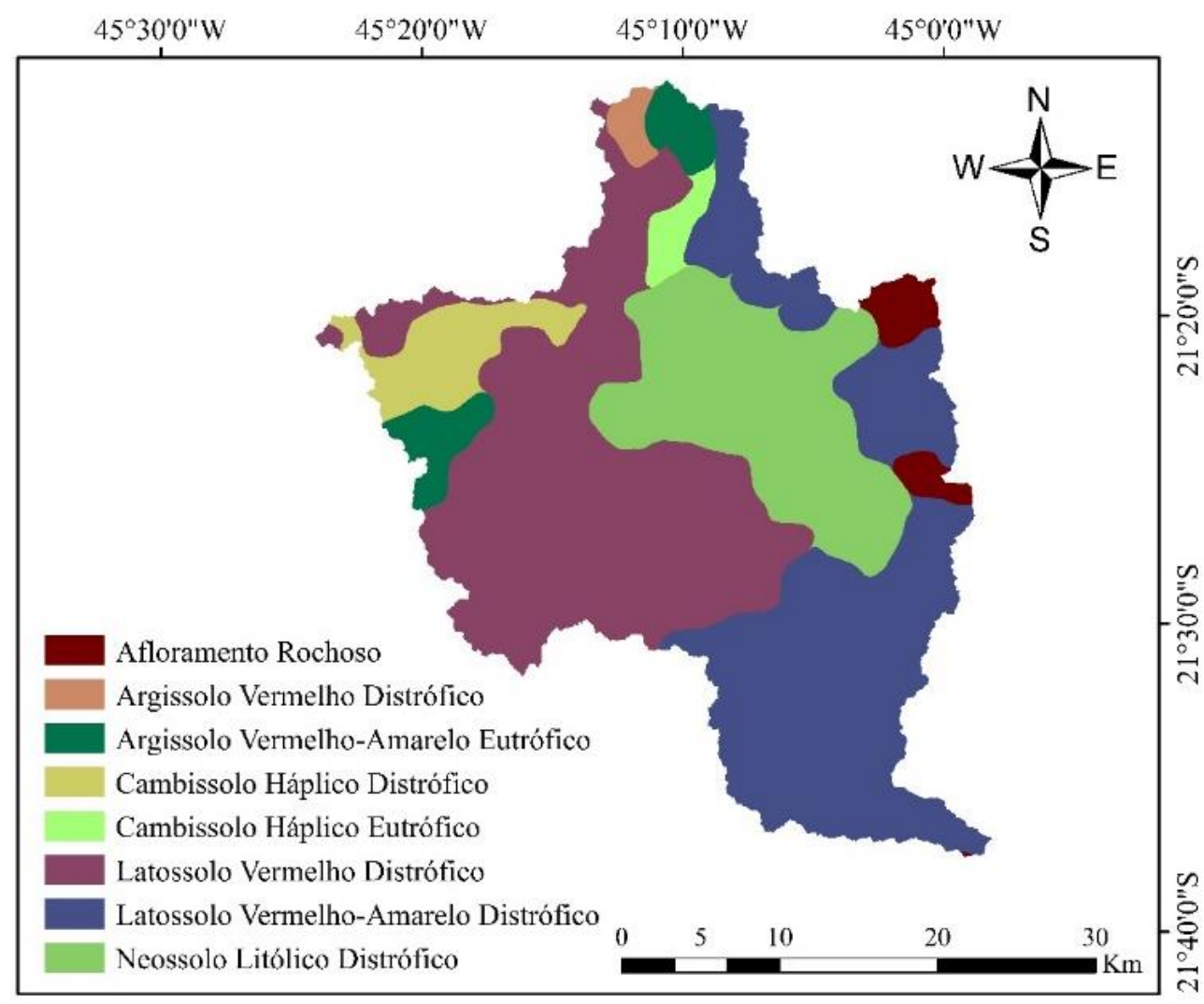

Figura 3 - Mapa de Solos da bacia hidrográfica do Rio Cervo, Minas Gerais.

Fonte: (Feam, 2010).

Tabela 2 - Valores de erodibilidade (K) para os diferentes tipos de solo.

\begin{tabular}{c|c|c}
\hline TIPO DE SOLO & K (t h MJ-1 mm-1) & FONTE \\
\hline ARGISSOLO VERMELHO distrófico - PVd & 0,032 & (Sá et al., 2004) \\
\hline ARGISSOLO VERMELHO-AMARELO eutrófico - PVAd & 0,033 & (Sá et al., 2004) \\
\hline CAMBISSOLO HÁPLICO distrófico - CXd & 0.024 & (Silva, 2003) \\
\hline CAMBISSOLO HÁPLICO eutrófico - CXe & 0,044 & (Mannigel et al., 2002) \\
\hline LATOSSOLO VERMELHO distrófico - LVd & 0,006 & (Mannigel et al., 2002) \\
\hline LATOSSOLO VERMELHO-AMARELO distrófico - LVAd & 0,011 & (Mannigel et al., 2002) \\
\hline NEOSSOLO LITÓLICO distrófico - RLd & 0,057 & (Oliveira et al., 2014)
\end{tabular}

\section{Fator Topográfico (LS)}

A erosão hídrica é profundamente afetada tanto pela distância ao longo da qual se propaga o escoamento superficial (L) quanto pela declividade do terreno $(\mathrm{S})$. $\mathrm{O}$ fator topográfico (LS) representa a relação entre as perdas de solo em uma área com comprimento de rampa e declividade quaisquer $\mathrm{e}$ as perdas de solo correspondentes em uma parcela padrão, caracterizada por 22,13 metros de comprimento com 9\% de declive (Wischmeier \& Smith, 1978). Quanto mais longo e íngreme for o declive, maior é a erosão.

Para estimar o fator LS tendo-se uma abordagem da RUSLE, foi empregada a metodologia proposta por (Moore \& Burch, 1986; Engel, 2003), também aplicada por Durães \& Mello (2014) e Oliveira et al. (2015) 
(Eq. 2), na qual, para obter o fator topográfico sobre um terreno tridimensional, utiliza-se um modelo digital de elevação (MDE) combinado a procedimentos de cálculo via "Raster Calculator Tool", no ArcGIS. O MDE utilizado para cobrir a bacia do Rio Cervo (Figura 4), provem das imagens ASTER ("Advanced Spaceborne Thermal Emission and Reflection
Radiometer") e apresenta resolução de 30 metros.

$$
L S=\left(F A x\left(\frac{C S}{22,13}\right)\right)^{0,4} \cdot\left(\frac{\operatorname{sen}(S)}{0,0896}\right)^{1,3}
$$

Sendo, FA é o fluxo acumulado; CS é o tamanho da célula do MDE (m) (comprimento do escoamento), e S é o "slope" (declive), em radianos.

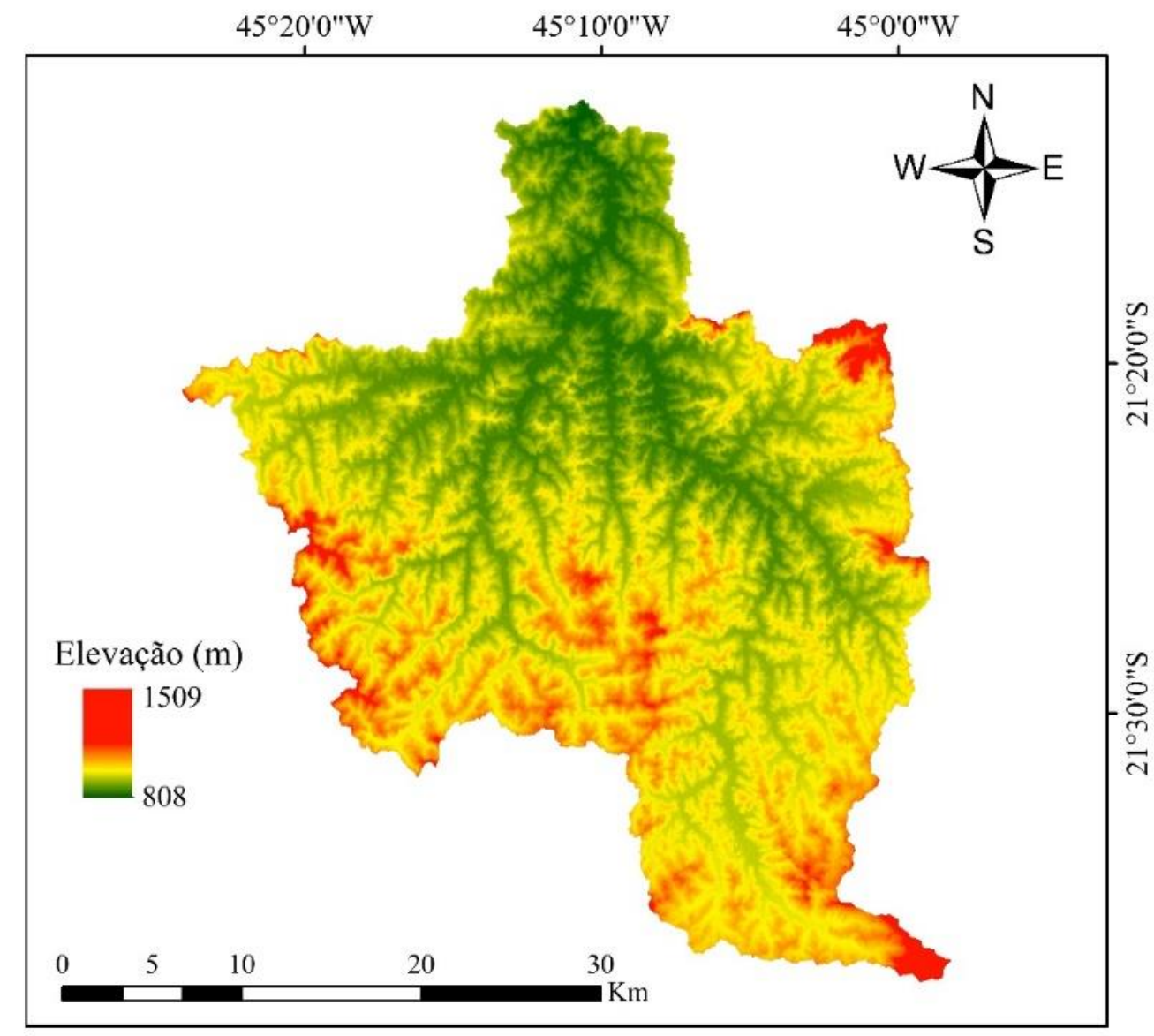

Figura 4 - Modelo Digital de Elevação da bacia hidrográfica do Rio Cervo, Minas Gerais.

\section{RESULTADOS E DISCUSSÃO}

Comportamento Espacial dos fatores passivos e ativos da erosão para a Bacia do Rio do Cervo, Sul de Minas Gerais

Os valores do fator $\mathrm{R}$ para a bacia do Rio Cervo variaram entre 6805 e 7684 MJ.mm.ha ${ }^{1} \cdot \mathrm{h}^{-1} \cdot$ ano $^{-1}$. Tais valores estão próximos aos estimados por Aquino et al. (2012) para a região Sul de Minas Gerais, onde verificou-se amplitude de 5.145 a $7.776 \mathrm{MJ} \cdot \mathrm{mm} \cdot \mathrm{ha}^{-1} \cdot \mathrm{h}^{-1}$.ano 1. Este intervalo de valores para o fator $\mathrm{R}$ pode ser classificado como "alto", de acordo com a classificação proposta por Foster et al. (1981).

$\mathrm{O}$ valor médio observado, calculado pixel a pixel, foi de $6866 \mathrm{MJ} \cdot \mathrm{mm} \cdot \mathrm{ha}^{-1} \cdot \mathrm{h}^{-1}$.ano ${ }^{-1}$, muito próximo ao encontrado por Val et al. (1986) para a cidade de Lavras, a qual está inserida na referida bacia (6837 MJ.mm.ha ${ }^{-1} \cdot h^{-1} \cdot$ ano $\left.^{-1}\right)$. Na Figura 5a está apresentado o mapa do fator $\mathrm{R}$ para a bacia do Rio Cervo, o qual permite um detalhamento espacial da erosividade em células de 30 metros.

Este mapa pode ser entendido como sendo um avanço metodológico importante em relação a outros estudos nos quais mapas do fator $\mathrm{R}$ foram desenvolvidos com base em poucas estações pluviométricas e uso de apenas uma ou duas equações para estimativa do Fator R com base no Índice Modificado de Fournier, não permitindo detalhar, por exemplo, efeitos de altitude nos valores do fator $\mathrm{R}$. 

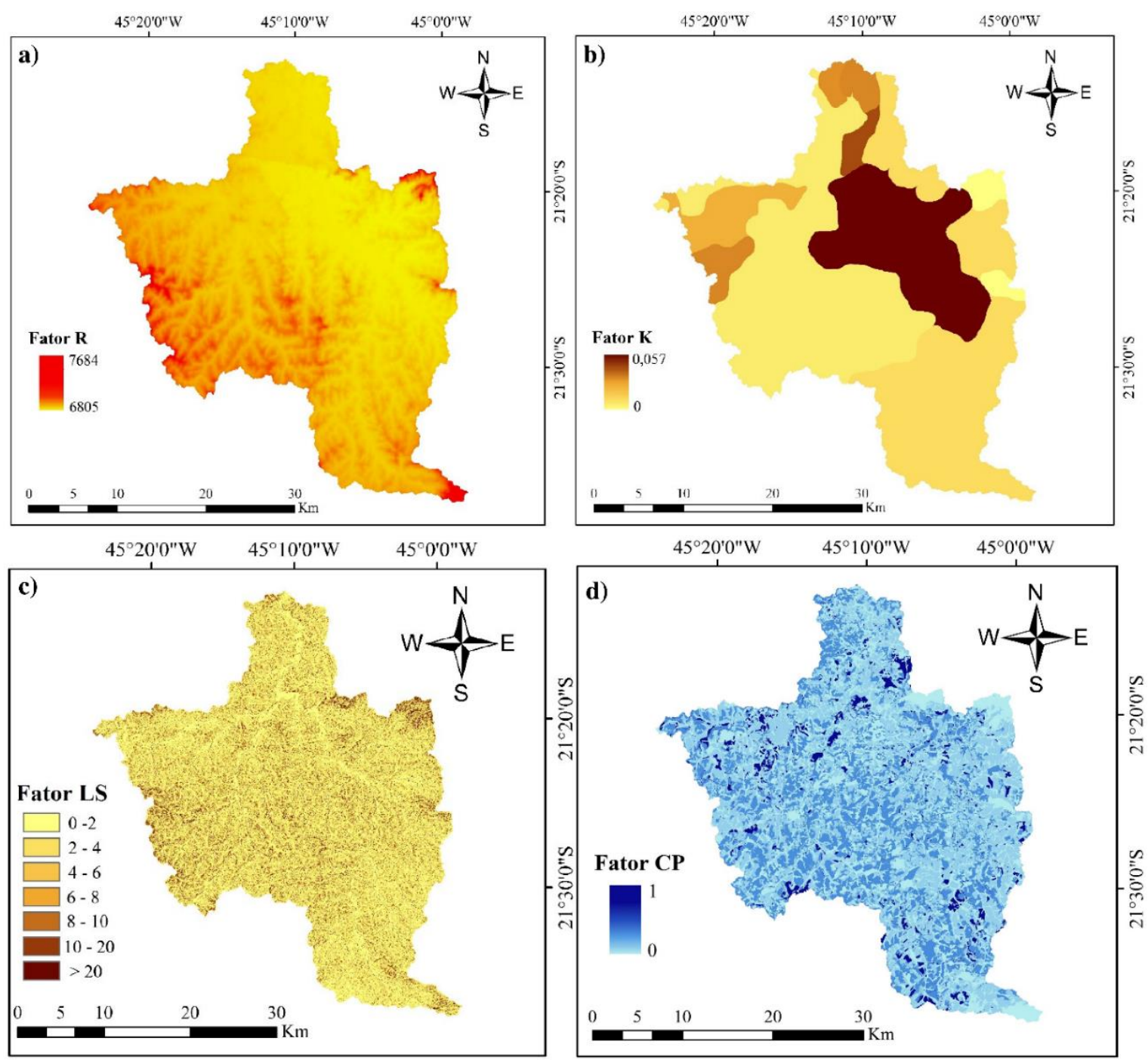

Figura 5 - Mapas dos fatores R (a), K (b), LS (c) e CP (d) da bacia hidrográfica do Rio Cervo - MG.

O mapa de erodibilidade do solo (Figura 5b) é um reflexo das unidades pedológicas presentes na área de estudo. $\mathrm{O}$ valor predominante do fator $\mathrm{K}$ na bacia do Rio Cervo foi de $0,006 \mathrm{t} \mathrm{h} \mathrm{MJ}^{-1} \mathrm{~mm}^{-1}$, correspondente ao Latossolo Vermelho distrófico $\quad(35,97 \%$ da área), sendo esta uma erodibilidade classificada como "muito baixa", segundo Bertoni \& Lombardi Neto (1999). Na sequência, aparece o valor de $0,011 \mathrm{t} \mathrm{h} \mathrm{MJ}^{-1} \mathrm{~mm}^{-1}$, correspondente ao Latossolo Vermelho-Amarelo distrófico (31,28\% da área), classificado como "baixo"; $0,057 \mathrm{t} \mathrm{h} \mathrm{MJ}^{-1} \mathrm{~mm}^{-1}$, correspondente ao Neossolo Litólico distrófico (18,84\% da área), considerado como de erodibilidade "muito alta"; 0,024 t h MJ ${ }^{-1} \mathrm{~mm}^{-1}$, para o Cambissolo Háplico distrófico $(5,15 \%$ da área), classificado como "moderada"; $0,033 \mathrm{t} \mathrm{h} \mathrm{MJ}^{-1} \mathrm{~mm}^{-1}$, correspondente ao Argissolo VermelhoAmarelo eutrófico $(3,85 \%$ da área), considerado como de erodibilidade "alta";
$0,044 \mathrm{t} \mathrm{h} \mathrm{MJ} \mathrm{mm}^{-1}$, correspondente ao Cambissolo Háplico eutrófico (1,47\% da área), considerado como "multo alta"; e $0,032 \mathrm{t} \mathrm{h} \mathrm{MJ}^{-}$ ${ }^{1} \mathrm{~mm}^{-1}$, correspondente ao Argissolo Vermelho distrófico $(0,97 \%$ da área), considerado como erodibilidade "alta".

Como os Latossolos ocupam $67 \%$ da área da bacia e estes devido às suas características pedológicas apresentam-se como de baixa vulnerabilidade à erosão, entende-se que a bacia do rio do Cervo, predominantemente, apresenta o fator $\mathrm{K}$ classificado como de "baixa" susceptibilidade à erosão.

A análise do fator LS é muito importante na configuração da erosão hídrica, uma vez que caracteriza a velocidade do escoamento superficial. A figura 5c representa o mapa do fator LS da bacia do rio do Cervo e a tabela 3, a distribuição das áreas em relação a cada intervalo do fator LS. Pode-se observar que quase $92 \%$ dos valores de LS presentes na bacia 
são menores do que 10 , o que representa uma vulnerabilidade moderada a erosão.

Resultado similar foi encontrado por Oliveira et al. (2014) para a bacia do rio Verde, também localizada na região sul do estado de Minas Gerais, onde foi observado $93 \%$ dos valores de LS menores do que 10.

Por outro lado, os $8 \%$ restantes demostram uma elevada vulnerabilidade a erosão, estando presentes nas áreas com maior declividade, onde o fluxo superficial apresenta-se com maior energia.

Tabela 3 - Distribuição do fator LS para a área de estudo.

\begin{tabular}{c|c|c|c}
\hline INTERVALO DO LS & ÁREA $\left.\mathbf{( k m}^{\mathbf{2}}\right)$ & ÁREA (\%) & ÁREA ACUMULADA (\%) \\
\hline $\mathbf{0 - 2}$ & 559,3 & 53,1 & 53,1 \\
\hline $\mathbf{2}-\mathbf{4}$ & 158,6 & 15,0 & 68,1 \\
\hline $\mathbf{4 - 6}$ & 124,9 & 11,9 & 80,0 \\
\hline $\mathbf{6 - 8}$ & 77,8 & 7,4 & 87,4 \\
\hline $\mathbf{8 - 1 0}$ & 45,6 & 4,3 & 91,7 \\
\hline $\mathbf{1 0 - 2 0}$ & 69,7 & 6,6 & 98,3 \\
\hline $\mathbf{2 0}$ & 18,1 & 1,7 & 100 \\
\hline
\end{tabular}

Na Figura 6 está apresentado o mapa de uso e manejo do solo referente ao ano de 2015 derivado das imagens "Rapideye" e na Tabela 4, a distribuição das áreas em relação a cada uso. Constata-se que a maior parte da bacia do Rio Cervo encontra-se antropizada, com $72,77 \%$ da área partilhada entre área urbana, agricultura, eucalipto, pastagem e solo exposto; desta forma a maior parte da bacia sofreu algum tipo impacto o que contribuiu para estimativas mais elevadas da perda de solo. O mapa do fator CP pode ser observado na Figura 5d, onde o valor predominante é 0,09 , correspondente a $40,46 \%$ da área.

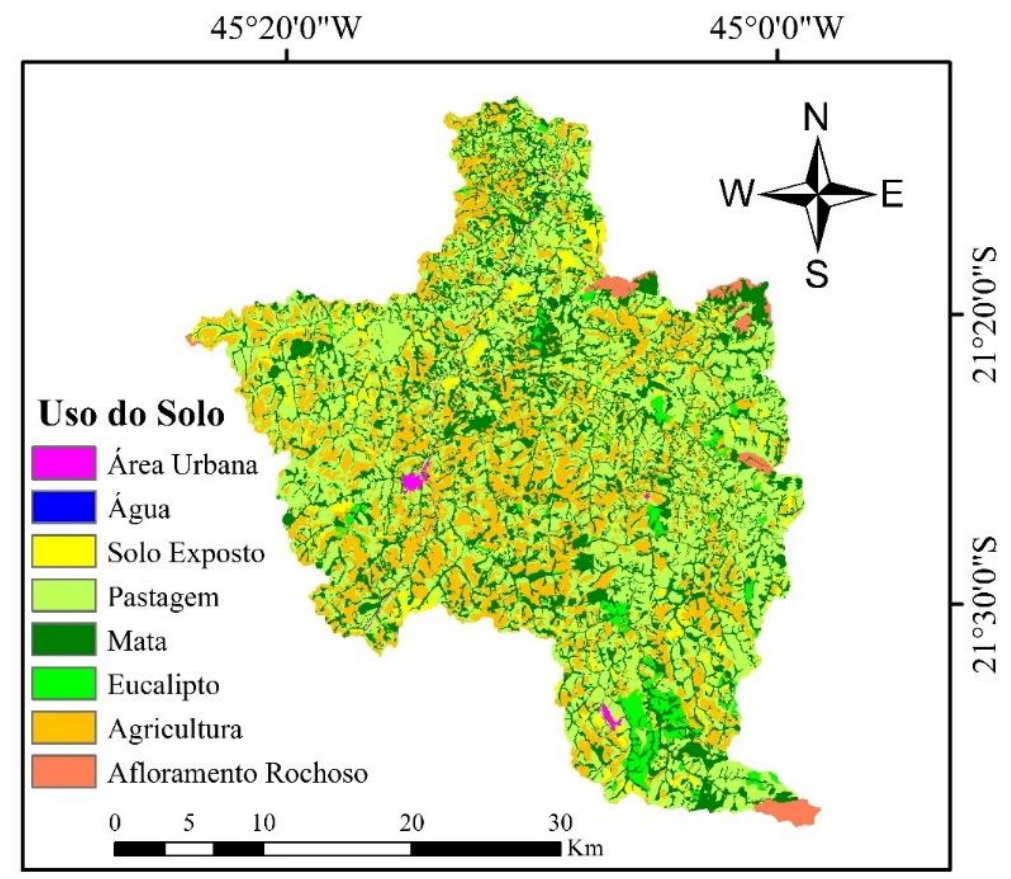

Figura 6. Mapa de uso do solo - 2015 da bacia hidrográfica do Rio Cervo - MG.

Tabela 4 - Distribuição das áreas em relação a cada uso.

\begin{tabular}{c|c|c}
\hline USO DO SOLO & ÁREA $\left.\mathbf{( k m}^{\mathbf{}}\right)$ & ÁREA $(\%)$ \\
\hline Área Urbana & 3,8 & 0,36 \\
\hline Água & 2,0 & 0,19 \\
\hline Afloramento Rochoso & 14,4 & 1,37 \\
\hline Agricultura & 259,6 & 24,63 \\
\hline Eucalipto & 27,7 & 2,63 \\
\hline Pastagem & 426,5 & 40,46 \\
\hline Mata & 270,6 & 25,67 \\
\hline Solo Exposto & 49,5 & 4,69 \\
\hline
\end{tabular}


Distribuição Espacial da taxa de erosão média anual para a Bacia do Rio do Cervo, Sul de Minas Gerais

A distribuição espacial da perda de solo para a bacia do Rio Cervo (Figura 7) foi obtida a partir da álgebra de mapas aplicada aos fatores da RUSLE. É importante salientar que devido as limitações da RUSLE, os valores estimados não podem ser tomados quantitativamente, devendo ser interpretados de forma qualitativa quanto ao potencial de ocorrência da erosão hídrica (Durães \& Mello, 2014; Barbosa et al., 2015).

Dessa forma, para a análise dos resultados adotou-se a classificação propostas por Beskow et al. (2009) a qual permite classificar a vulnerabilidade do solo à erosão desde "ligeira" a "extremamente alta".

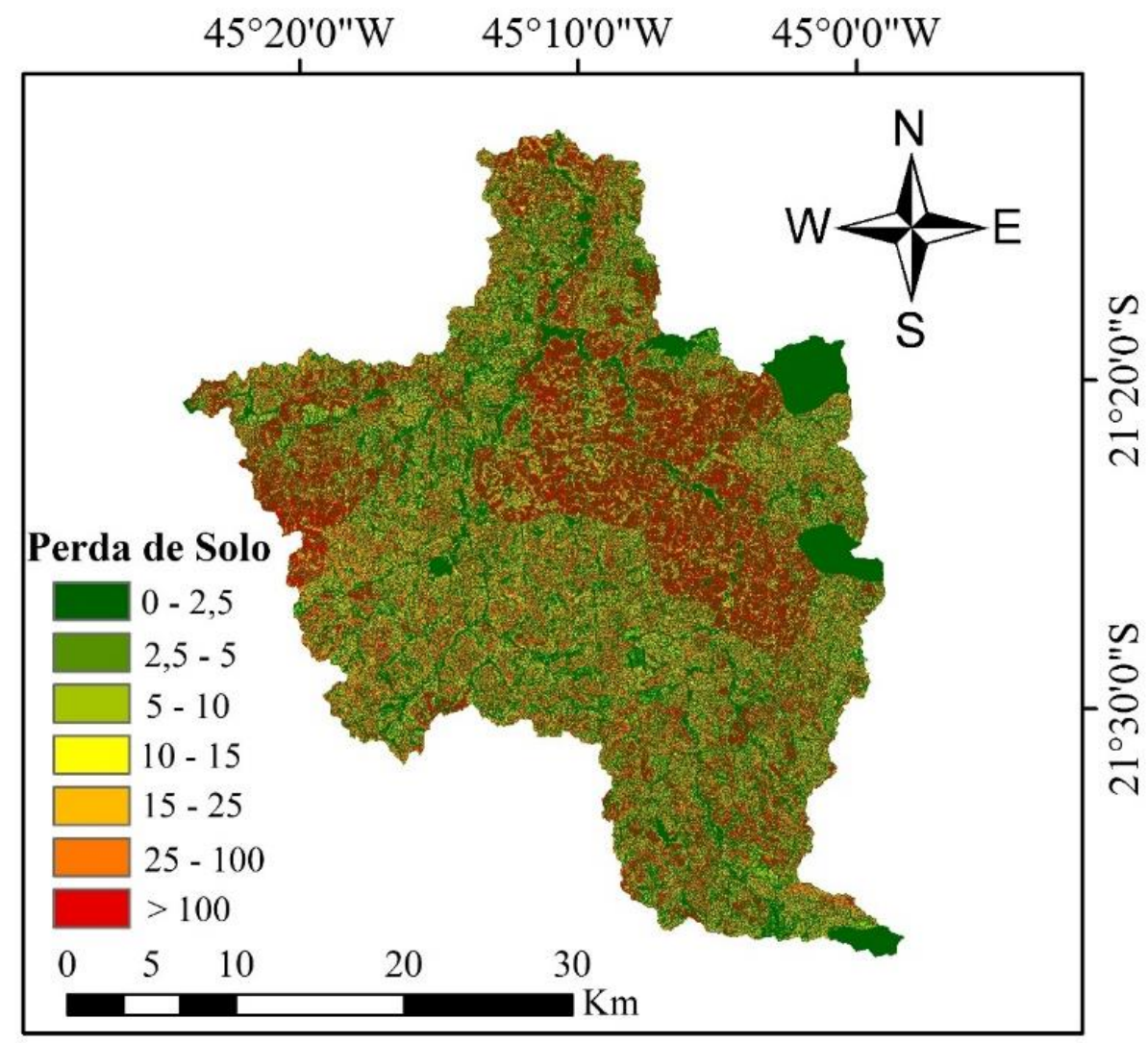

Figura 7 - Perda de solo $\left(\mathrm{t} \mathrm{ha}^{-1} \mathrm{ano}^{-1}\right)$ da bacia hidrográfica do Rio Cervo - MG.

$\mathrm{Na}$ tabela 5, pode-se observar que na maior parte de bacia $(50,83 \%)$ há predomínio da classe de vulnerabilidade "ligeira". Esse resultado pode ser explicado com base nos baixos valores do fator $\mathrm{K}$, uma vez que os Latossolos e afloramentos rochosos ocupam $69,72 \%$ da área, e também devido à presença de valores de CP inferiores a 0,01 em $27,59 \%$ da bacia, o qual significa boa proteção do solo a impactos das gotas de chuva. No entanto, $40,59 \%$ da bacia apresentou perdas de solo superiores a $10 \mathrm{t}$ $\mathrm{ha}^{-1} \mathrm{ano}^{-1}$, com predomínio da classe de vulnerabilidade "muito alta", o que indica a existência de áreas susceptíveis a processos erosivos críticos, demonstrando assim a necessidade da adoção de práticas de conservacionistas.

Tabela 5 - Classificação da vulnerabilidade a erosão da bacia hidrográfica do Rio Cervo - MG.

\begin{tabular}{c|c|c}
\hline PERDA DE SOLO $\left(\mathbf{t ~ h a}^{-\mathbf{1}} \mathbf{a n o}^{-\mathbf{1}}\right)$ & VULNERABILIDADE & ÁREA (\%) \\
\hline $\mathbf{0}-\mathbf{2 , 5}$ & Ligeira & 50,83 \\
\hline $\mathbf{2 , 5}-\mathbf{5}$ & Ligeira a Moderada & 4,04 \\
\hline $\mathbf{5}-\mathbf{1 0}$ & Moderada & 4,54 \\
\hline $\mathbf{1 0}-\mathbf{1 5}$ & Moderada a Alta & 3,69 \\
\hline $\mathbf{1 5}-\mathbf{2 5}$ & Alta & 6,13 \\
\hline $\mathbf{2 5}-\mathbf{1 0 0}$ & Muito Alta & 17,12 \\
\hline $\mathbf{1 0 0}$ & Extremamente Alta & 13,65 \\
\hline
\end{tabular}


Analisando a distribuição espacial das nascentes da bacia do Rio Cervo (Figura 8); extraída a partir de procedimentos de cálculo aplicados ao MDE, no âmbito da taxa de erosão média anual, pode-se observar que de um total de 621 nascentes identificadas, $41,7 \%$ estão situadas em áreas classificadas como "ligeira" vulnerabilidade a erosão; 9,7\% situadas em áreas classificadas como "ligeira a moderada"; $8,1 \%$ na classe "moderada"; $5 \%$ na classe "moderada a alta"; 4,3\% na classe "alta"; $17,4 \%$ na classe "muito alta" e $13,8 \%$ na classe "extremamente alta".

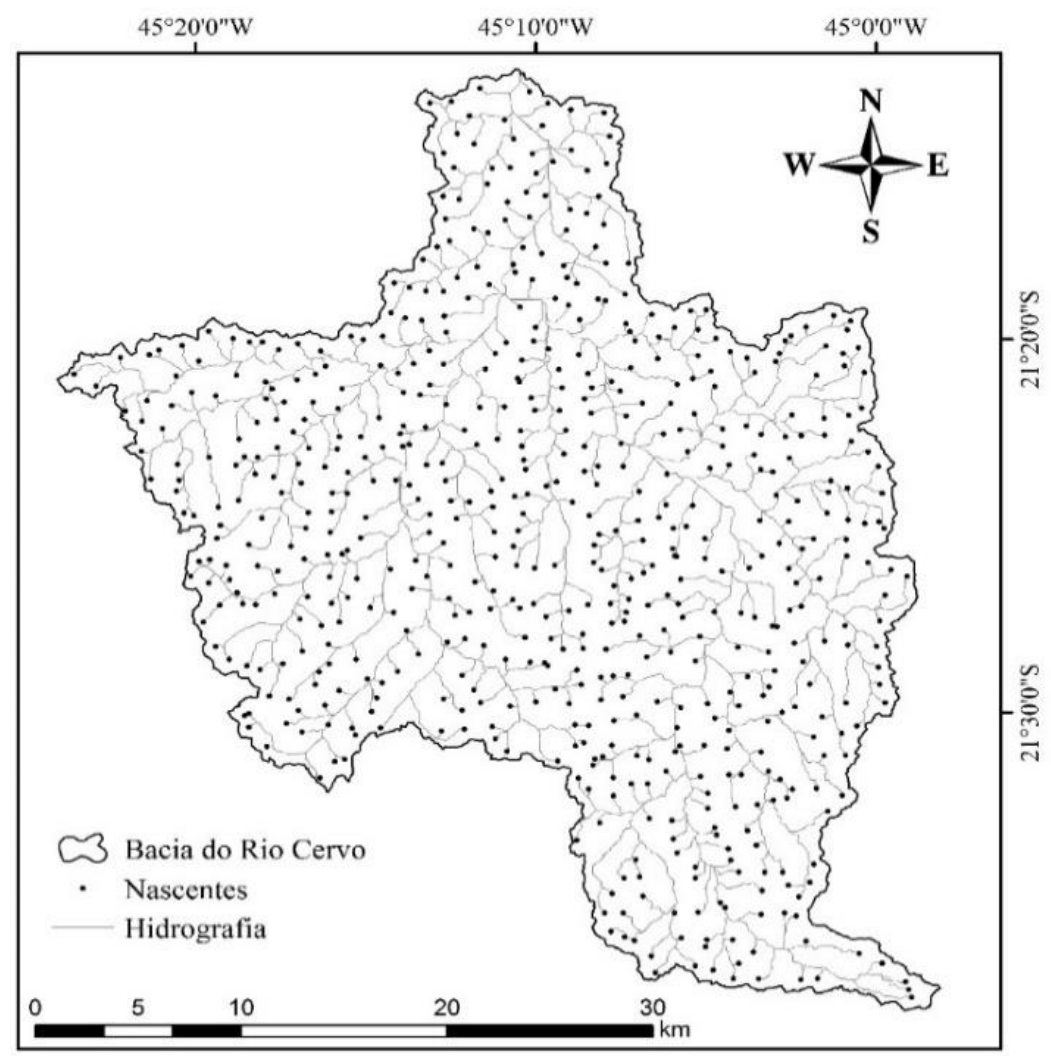

Figura 8 - Distribuição espacial das nascentes da bacia hidrográfica do Rio Cervo - MG.

Esses valores demonstram que $35,6 \%$ das nascentes estão localizadas em áreas que apresentam perdas de solo superiores a $15 \mathrm{t} \mathrm{ha}^{-1}$ ano $^{-1}$, podendo este resultado ser explicado pelo fato de $48 \%$ destas estarem na presença de solo exposto e pastagem, $25 \%$ estarem na presença de agricultura e eucalipto e $29 \%$ estarem situadas na unidade pedológica Neossolo Litólico distrófico. Dessa forma, verifica-se que a alta taxa de erosão que atinge as áreas de nascentes é predominantemente consequência da gestão inadequada dos recursos naturais e o não cumprimento do Código Florestal Brasileiro, Lei Federal 12.651/2012 (Brasil, 2012), onde proíbe-se o desmatamento da área imediatamente circundante à nascente, em um raio de 50 metros.

Na tabela 6, pode-se observar os valores de perda de solo para cada intervalo de vulnerabilidade a erosão e para cada unidade pedológica. O Neossolo Litólico foi responsável por grande parte da vulnerabilidade "extremamente alta" da bacia, sendo que dos $13,65 \%$ identificados, este contribuiu com $6,11 \%$. Esse comportamento pode ser explicado pela combinação do seu elevado fator $\mathrm{K}$ com um uso do solo predominante de pastagem. As perdas de solo em Latossolos foram responsáveis por $67,25 \%$ das perdas totais, no entanto, $34,6 \%$ dessas perdas ocorreram na classe de vulnerabilidade "ligeira".

A fim de analisar as perdas de solo em termos de tolerância de cada unidade pedológica, foi extraído dos estudos desenvolvidos por Demarchi (2012), Mannigel et al. (2002) e Silva (2003), os seguintes valores de tolerância de perda do solo, dados em $\mathrm{t} \mathrm{ha}^{-1}$ ano $^{-1}: 9,11 ; 9,06 ; 11,62 ; 3,67 ; 11,34 ; 11,53 ; 6,8$, para PVd, PVAe, CXe, CXd, LVd, LVAd, RLd, respectivamente. Comparando esses valores com a tabela 6 , pode-se desenvolver um gráfico (Figura 9) onde está apresentado a 
porcentagem da área da bacia com maiores perdas de solo bem como o percentual das áreas com erosão abaixo do limite máximo tolerável para cada tipo de solo. Atualmente, $41 \%$ da área total da bacia tem perda de solo superior à taxa tolerável, indicando assim a existência de processos críticos de erosão e deficiência na gestão de uso da terra na bacia hidrográfica.

Tabela 6 - Variação da perda de solo (\%) para diferentes tipos de solos na bacia hidrográfica do Rio Cervo - MG.

\begin{tabular}{|c|c|c|c|c|c|c|c|c|}
\hline \multirow{2}{*}{ Tipo de Solo } & \multicolumn{8}{|c|}{ Intervalos de perda de solo $\left(\mathrm{t} \mathrm{ha}^{-1} \mathrm{ano}^{-1}\right)$} \\
\hline & $0-2,5$ & $2,5-5$ & $5-10$ & $10-15$ & $15-25$ & 25-100 & $>100$ & Total (\%) \\
\hline $\mathbf{A R}$ & 2,47 & 0,00 & 0,00 & 0,00 & 0,00 & 0,00 & 0,00 & 2,47 \\
\hline PVd & 0,40 & 0,03 & 0,06 & 0,04 & 0,06 & 0,13 & 0,25 & 0,97 \\
\hline PVAe & 1,69 & 0,07 & 0,13 & 0,11 & 0,14 & 0,58 & 1,13 & 3,85 \\
\hline CXe & 0,81 & 0,02 & 0,05 & 0,04 & 0,06 & 0,18 & 0,31 & 1,47 \\
\hline CXd & 2,44 & 0,15 & 0,25 & 0,16 & 0,20 & 0,92 & 1,03 & 5,15 \\
\hline LVd & 18,34 & 1,87 & 1,91 & 1,65 & 2,78 & 7,35 & 2,07 & 35,97 \\
\hline LVAd & 16,26 & 1,68 & 1,62 & 1,15 & 2,01 & 5,81 & 2,75 & 31,28 \\
\hline RLd & 8,42 & 0,22 & 0,52 & 0,54 & 0,88 & 2,15 & 6,11 & 18,84 \\
\hline Total (\%) & 50,83 & 4,04 & 4,54 & 3,69 & 6,13 & 17,12 & 13,65 & 100 \\
\hline
\end{tabular}

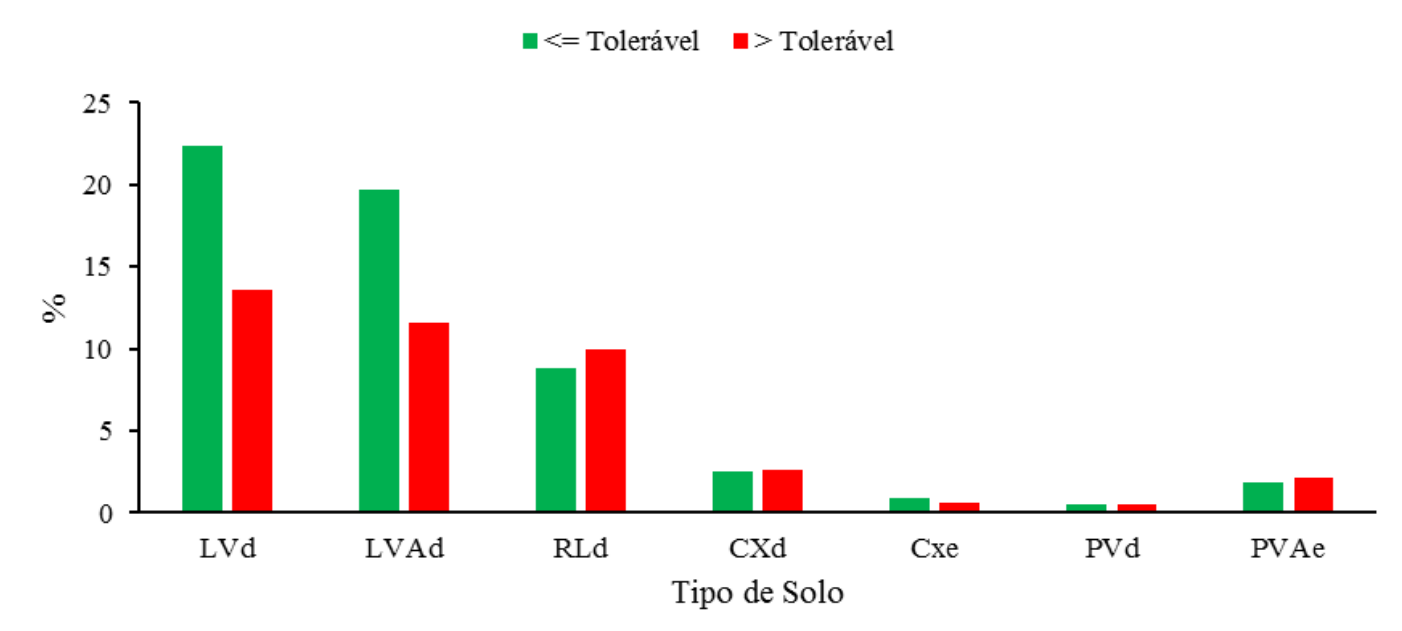

Figura 9 - Porcentagem da área da bacia com perdas de solo maior e menos ao tolerável para cada tipo de solo.

\section{CONCLUSÕES}

Os resultados mostraram que a utilização do modelo empírico RUSLE associado a um SIG, apesar das limitações no aspecto quantitativo, apresenta-se como uma ferramenta eficaz na estimativa da vulnerabilidade a erosão dos solos, permitindo identificar as taxas de erosão hídrica resultantes da utilização atual do solo e como ferramenta para subsidiar serviços ecológicos visando à sustentabilidade. Além disso, pode-se verificar que parte considerável da bacia do Rio Cervo (50,83\% de sua área), apresenta "ligeira" vulnerabilidade à erosão, em contrapartida, $40,59 \%$ apresenta elevada vulnerabilidade, indicando assim a necessidade de implementar práticas de conservação do solo para reduzir a vulnerabilidade nessas áreas.

\section{AGRADECIMENTOS}

Os autores agradecem à CAPES pela concessão da bolsa de mestrado à primeira autora, e ao CNPq pelo apoio financeiro ao projeto 482075/2013-9 e bolsa de produtividade em pesquisa ao terceiro autor (305854/2015-1).

\section{REFERÊNCIAS}

AMARAL, A.J. DO. Fator cobertura e manejo da Equação Universal de Perda de Solo para soja e trigo em um Cambissolo Húmico Alumínico submetido a diferentes sistemas de manejo. Lages, 2006. 112 p. Dissertação (Mestrado em Ciências do Solo)-Universidade do Estado de Santa Catarina.
AQUINO, R.F.; SILVA, M.L.N.; FREITAS, D.A. F.; CURI, N.; MELLO, C.R.; AVANZI, J.C. Spatial variability of the rainfall erosivity in southern region of Minas Gerais State, Brazil. Ciência e Agrotecnologia, v. 36, n. 5, p. 533-542, 2012. 
AYER, J.E.B.; OLIVETTI, D.; MINCATO, R.L.; SILVA, M.L.N. Erosão hídrica em Latossolos Vermelhos distróficos. Pesquisa Agropecuária Tropical, v. 45, n. 2, p.180-191, 2015.

BARBOSA, A. F.; OLIVEIRA, E. F.; MIOTO, C. L.; PARANHOS FILHO, A. C. Aplicação da Equação Universal de Perda do Solo (USLE) em Softwares Livres e Gratuitos. Anuário do Instituto de Geociências - UFRJ. v. 38, p. 170179, 2015.

BERTONI, J. \& LOMBARDI NETO, F. Conservação do solo. 2.ed. São Paulo, Ícone, 355p., 1999.

BESKOW, S.; MELLO, C.R.; NORTON, L.D.; CURI, N.; VIOLA, M.R.; AVANZI, J.C. Soil erosion prediction in the Grande River Basin, Brazil using distributed modeling. Catena. v. 79, n. 1, p. 49-59, 2009.

BONILLA, C. A; REYES, J. L.; MAGRI, A. Water Erosion Prediction Using the Revised Universal Soil Loss Equation (RUSLE) in a GIS Framework, Central Chile. Chilean Journal of Agricultural Research. v. 70, n. 1, p. 159-169, 2010.

BRASIL. Lei $\mathbf{N}^{\mathbf{0}} \mathbf{1 2 . 6 5 1}$, de 25 de maio de 2012. Institui o novo Código Florestal. Diário Oficial da União, Brasília, 2012. Disponível em: http://www.planalto.gov.br /ccivil_03/_Ato2011-2014/2012/Lei/L12651.htm. Acessado em: 05 abr 2016.

CAPOANE, V. Utilização do fator topográfico da RUSLE para análise da susceptibilidade a erosão do solo em uma bacia hidrográfica com pecuária intensiva do sul do Brasil. Revista Geonorte, v.8, p. 85-101, 2013.

DEMARCHI, J.C. Geotecnologias aplicadas à estimativa de perdas de solo por erosão hídrica na Sub-Bacia do Ribeirão das Perobas, município de Santa Cruz do Rio Pardo-SP. Botucatu, 2012. 167 p. Dissertação (Mestrado em Agronomia) - Universidade Estadual Paulista.

DIAS, E.R. \& SILVA, R.M. Estimativa do risco à erosão do solo no município de Lucena - Paraíba. Caminhos de Geografia. v. 16, n. 54, p. 192-204, 2015.

DURÃES, M.F. \& MELLO, C.R. Hydrosedimentologic disturbance index applied to watersheds of Minas Gerais state. Ciência \& Agrotecnologia, v. 38, n. 1, p. 61-67, 2014.

ENGEL, B. Estimating soil erosion using RUSLE - Using Arcview. Purdue University, 2003. Disponível em: http://pasture.ecn.purdue.edu/ engelb/ agen526/gisrusle /gisrusle.html. Acessado em: 20 jan 2016.

FEAM - FUNDAÇÃO ESTADUAL DO MEIO AMBIENTE. Mapa de solos de Minas Gerais: legenda expandida. Belo Horizonte: FEAM/UFV/CETEC/UFLA. 49 p. 2010.

FJP - FUNDAÇÃO JOÃO PINHEIRO. Produto interno bruto das regiões de planejamento de Minas Gerais. 2009. Disponível em: http://www.fjp.mg.gov.br /índex.php/analiseeconomica/pib-produto-interno-bruto-de-minas- gerais. Acesso em: 10 fev 2016

FOSTER, G.R.; MCCOOL, D.K.; RENARD, K.G.; MOLDENHAUER, W. C. Conversion of the universal soil loss equation to SI metric units. Journal of Soil and Water Conservation. v. 36, p. 355- 359, 1981.

KINNELL, P.I.A. Event soil loss, runoff and the Universal Soil Loss Equation family of models: a review. Journal of Hydrology, v. 385, n. 1, p. 384-397, 2010.

MANNIGEL, A.R.; CARVALHO, M.P.; MORETI, D.; MEDEIROS, L.R. Fator erodibilidade e tolerância de perda dos solos do Estado de São Paulo. Acta Scientiarum, v. 24, n. 5, p. 1335-1340, 2002.

MARTINS, S.G.; SILVA, M.L.N.; AVANZI, J.C.; CURI, N.; FONSECA, S. Fator cobertura e manejo do solo e perdas de solo e água em cultivo de eucalipto e em Mata Atlântica nos Tabuleiros Costeiros do estado do Espírito Santo. Scientia Forestalis, v. 38, n. 87, p. 517-526, 2010.
MELLO, C. R.; VIOLA, M. R.; BESKOW, S.; NORTON, L; D. Multivariate models for annual rainfall erosivity in Brazil. Geoderma, v. 202-203, p. 88-102, 2013.

MITASOVA, H.; BROWN, W. N; JOHNSTON, D.M. Terrain Modeling and Soil Erosion Simulation Final Report. Champaign: University of Illinois, 2001. Disponível em: http://www4.ncsu.edu/ hmitaso/gmslab/reports/cerl01/finalre port/report01/default.htm. Acessado em: 20 nov 2015.

MOORE, I.D. \& BURCH, G.J. Modeling erosion and deposition: Topographic effects. Transactions of the America, Science Agricultural Engineering, v. 29, n. 6, p. 1624-1640, 1986.

OLIVEIRA, V.A.; MELLO, C.R.; DURÃES, M.F.; SILVA, A. M. Soil erosion vulnerability in the Verde River Basin, Southern Minas Gerais. Ciência \& Agrotecnologia, v. 38, n. 3, p. 262-269, 2014.

OZSOY, G.; AKSOY, E.; DIRIM, M.S.; TUMSAVAS, Z. Determination of Soil Erosion Risk in the Mustafakemalpasa River Basin, Turkey, Using the Revised Universal Soil Loss Equation, Geographic Information System, and Remote Sensing. Environmental Management, v. 50, p. 679-694, 2012.

PANAGOS, P.; BORRELI, P.; POESEN, J.; BALLABIO, C.; LUGATO, E.; MEUSBURGER, K.; MONTANARELLA, L.; ALEWELL, C. The new assessment of soil loss by water erosion in Europe. Environmental Science \& Policy, v. 54, p. 438-447, 2015.

PANDEY, A.; CHOWDARY, V. M.; MAL, B.C. Identification of critical erosion prone areas in the small agricultural watershed using USLE, GIS and remote sensing. Water Resour Manage, v. 21, p. 729-746, 2007.

RENARD, K.G.; FOSTER, G.R.; WEESIES, G.A.; MCCOOL, D.K.; YODER, D.C. Predicting soil erosion by water: a guide to conservation planning with the Revised Universal Soil Loss Equation (RUSLE). Agriculture Handbook. Washington, DC: USDA, v. 703, 384 p., 1997.

RUHOFF, A.L.; SOUZA, B.S.P.; GIOTTO, E.; PEREIRA, R.S. Avaliação dos processos erosivos através da Equação Universal de Perda de Solos, implementada com algoritmos em LEGAL. Geomática, v. 1, n. 1, p. 12-22, 2006.

SÁ, M.A.C.; LIMA, J. M.; CURI, N.; MASSAROTO, J.A.; MARQUES, J.J.G.S.M. Estimativa da erodibilidade pela desagregação por ultra-som e atributos de solos com horizonte B textural. Pesquisa Agropecuária Brasileira, v. 39, n. 7, p. 691-699, 2004.

SÁ JÚNIOR, A. Aplicação da classificação de Köppen para o zoneamento climático do Estado de Minas Gerais. Lavras, 2009. 113p. Dissertação (Mestrado em Engenharia Agrícola) - Universidade Federal de Lavras.

SHIFERAW, A. Estimating soil loss rates for soil conservation planning in the Borena Woreda of South Wollo Highlands, Ethiopia. Journal of Sustainable Development in Africa, v. 13, n. 3, p. 87-106, 2011.

SILVA, A.M. Erosividade, erodibilidade e perdas por erosão em Cambissolo e Latossolo sob chuva natural. Lavras, 2003. 72 p. Dissertação (Mestrado em Solos e Nutrição de Plantas) - Universidade Federal de Lavras.

VAL, L.A.; BAHIA, V.G.; FREIRE, J.C.; DIAS JUNIOR, M.S. Erosividade das chuvas em Lavras, MG. Ciência e Prática. v. 10, n. 2, p. 199-209, 1986.

WISCHMEIER, W.H. \& SMITH, D.D. Predicting rainfall erosion losses: A guide to conservation planning. Agriculture Handbook. Washington, DC: USDA, v. 537, 58 p., 1978.

Submetido em 19 de agosto de 2016 Aceito em 21 de dezembro de 2016 